PROCEEDINGS OF THE

AMERICAN MATHEMATICAL SOCIETY

Volume 131, Number 10, Pages 3003-3007

S 0002-9939(03)07167-3

Article electronically published on May 9, 2003

\title{
LOCAL RINGS OF COUNTABLE COHEN-MACAULAY TYPE
}

\author{
CRAIG HUNEKE AND GRAHAM J. LEUSCHKE
}

(Communicated by Bernd Ulrich)

\begin{abstract}
We prove (the excellent case of) Schreyer's conjecture that a local ring with countable $\mathrm{CM}$ type has at most a one-dimensional singular locus. Furthermore, we prove that the localization of a Cohen-Macaulay local ring of countable CM type is again of countable CM type.
\end{abstract}

Let $(R, \mathfrak{m})$ be a (commutative Noetherian) local ring of dimension $d$. Recall that a nonzero $R$-module $M$ is called maximal Cohen-Macaulay (MCM) provided it is finitely generated and there exists an $M$-regular sequence $\left\{x_{1}, \ldots, x_{d}\right\}$ in the maximal ideal $\mathfrak{m}$. We say that $R$ itself is Cohen-Macaulay (CM) if it is MCM as a module over itself.

The CM local rings of finite CM-representation type (meaning that they have only finitely many nonisomorphic indecomposable MCM modules) have been carefully studied over the last twenty years. The complete equicharacteristic hypersurfaces of finite CM type have been completely classified ([6], [4, 9]), as have the complete equicharacteristic 2-dimensional normal domains (2]). More generally, it is known that a CM local ring of finite CM type has at most an isolated singularity (proved by Auslander [1] in the complete case, Leuschke-Wiegand [10] in the excellent case, and Huneke-Leuschke [8] in general). Yoshino's monograph [15] is a comprehensive source for information about rings of finite CM type.

The related property of countable CM type has received much less attention. Buchweitz, Greuel, and Schreyer [4] classified the complete hypersurface singularities of countable CM type, but not much more has been learned since then.

The open questions and conjectures in Schreyer's 1987 survey article 12 have inspired work on both finite and countable CM-representation type. For example, Conjecture 7.3(a) states that a CM local ring $R$ has finite CM type if and only if the $\mathfrak{m}$-adic completion has finite CM type; this was recently proved in case $R$ is excellent in 10. This paper is concerned with another of Schreyer's conjectures:

Conjecture $0.1([12])$. An analytic local ring over the complex numbers of countable $C M$ type has at most a one-dimensional singular locus, that is, $R_{\mathfrak{p}}$ is regular for all primes $\mathfrak{p}$ with $\operatorname{dim} R / \mathfrak{p}>1$.

We verify Conjecture 0.1 more generally for all excellent CM local rings satisfying countable prime avoidance (Lemma 1.2). Some assumption of uncountability is

Received by the editors May 10, 2002.

2000 Mathematics Subject Classification. Primary 13C14; Secondary 13H10, 13 C05.

Key words and phrases. Maximal Cohen-Macaulay modules, CM representation type, countable CM type.

Both authors were supported by the National Science Foundation. 
necessary to avoid the degenerate case of a countable ring, which a fortiori has only countably many isomorphism classes of modules.

\section{SCHREyer's CONJECTURE}

Definition 1.1. A Cohen-Macaulay local $\operatorname{ring}(R, \mathfrak{m})$ is said to have finite (resp., countable) Cohen-Macaulay type if it has only finitely (resp., countably) many isomorphism classes of maximal Cohen-Macaulay modules.

For the proof of Schreyer's conjecture, we need the following well-known lemma.

Lemma 1.2 (countable prime avoidance [5, Lemma 3]; see also [13]). Let $A$ be a Noetherian local ring which either is complete or has uncountable residue field. Let $\left\{\mathfrak{p}_{i}\right\}, i=1,2, \ldots$, be a countable family of prime ideals of $A, \mathfrak{a}$ an ideal of $A, x \in A$. Then $x+\mathfrak{a} \subseteq \mathfrak{p}_{j}$ for some $j$ whenever $x+\mathfrak{a} \subseteq \bigcup_{i=1}^{\infty} \mathfrak{p}_{i}$.

Theorem 1.3. Let $(R, \mathfrak{m})$ be an excellent Cohen-Macaulay local ring of dimension $d$, and assume either that $R$ is complete or that the residue field $R / \mathfrak{m}$ is uncountable. If $R$ has countable CM type, then the singular locus of $R$ has dimension at most one.

Proof. Assume that the singular locus of $R$ has dimension greater than one. Since $R$ is excellent, this means that the singular locus is defined by an ideal $J$ of height strictly less than $d-1$. Let $\left\{M_{i}\right\}_{i=1}^{\infty}$ be a complete list of representatives for the isomorphism classes of indecomposable MCM $R$-modules. Consider the set

$$
\Lambda=\left\{\mathfrak{p} \in \operatorname{Spec}(R) \mid \mathfrak{p}=\operatorname{Ann}\left(\operatorname{Ext}_{R}^{1}\left(M_{i}, M_{j}\right)\right) \text {, for some } i, j, \text { and } \operatorname{dim}(R / \mathfrak{p})=1\right\} .
$$

Note that $\Lambda$ is at most countable, and that $J$ is contained in each $\mathfrak{p} \in \Lambda$. By countable prime avoidance (applied to $R / J$ ), the maximal ideal $\mathfrak{m}$ is not contained in the union of all $\mathfrak{p}$ in $\Lambda$, so there is an element $f \in \mathfrak{m} \backslash \bigcup_{\mathfrak{p} \in \Lambda} \mathfrak{p}$. Choose a prime $\mathfrak{q}$ containing $f$ and $J$ such that $\operatorname{dim} R / \mathfrak{q}=1$; then, of course, $\mathfrak{q} \notin \Lambda$.

Let $X($ resp. $Y)$ be a $(d-1)^{\text {th }}\left(\right.$ resp. $\left.d^{\text {th }}\right)$ syzygy of $R / \mathfrak{q}$. Then $X$ and $Y$ are both MCM $R$-modules and we have a nonsplit short exact sequence

$$
0 \longrightarrow Y \longrightarrow F \longrightarrow X \longrightarrow 0,
$$

where $F$ is a free $R$-module. We claim that $\operatorname{Ann}\left(\operatorname{Ext}_{R}^{1}(X, Y)\right)=\mathfrak{q}$. It is clear that $\mathfrak{q}$ kills $\operatorname{Ext}_{R}^{1}(X, Y) \cong \operatorname{Ext}_{R}^{d}(R / \mathfrak{q}, Y)$. To see the opposite containment, note that since $\mathfrak{q}$ contains $J, R_{\mathfrak{q}}$ is not regular. The resolution of the residue field of $R / \mathfrak{q}$ is thus infinite, and neither $X_{\mathfrak{q}}$ nor $Y_{\mathfrak{q}}$ is free, so $(\circledast)$ is nonsplit when localized at $\mathfrak{q}$.

We can write both $X$ and $Y$ as direct sums of copies of the indecomposables $M_{i}$, and further write

$$
\operatorname{Ext}_{R}^{1}(X, Y) \cong \bigoplus_{i, j} \operatorname{Ext}_{R}^{1}\left(M_{i}, M_{j}\right)^{a_{i j}}
$$

with all but finitely many of the $a_{i j}$ equal to zero. Then $\mathfrak{q}$ is the intersection of the annihilators of the nonzero Ext modules appearing in the above decomposition. Since $\mathfrak{q}$ is prime, it must equal one of these annihilators, and then $\mathfrak{q} \in \Lambda$, a contradiction. 


\section{Localization of Rings With COUntable CM Type}

Let $(R, \mathfrak{m})$ be an excellent local ring of countable CM type, and assume $R$ either has an uncountable residue field or is complete. By Theorem [1.3, the dimension of the singular locus of $R$ is at most one. Thus there are at most finitely many prime ideals $\mathfrak{p}_{1}, \ldots, \mathfrak{p}_{n}$ such that $R_{\mathfrak{p}_{i}}$ is not regular and $\mathfrak{p}_{i} \neq \mathfrak{m}$. All such primes have dimension one, i.e., $\operatorname{dim}\left(R / \mathfrak{p}_{i}\right)=1$ for $i=1, \ldots, n$. To understand the structure of these rings, one wishes to know what type of singularity $R$ has at these primes. A quick inspection of the list of examples given in [12] shows that $R_{\mathfrak{p}_{i}}$ has finite CM type! Our next main result shows that countable CM type localizes; hence in general each $R_{\mathfrak{p}_{i}}$ has countable CM type. Note also that in the case $R$ is complete, while $R_{\mathfrak{p}_{i}}$ is no longer complete in general, it will have uncountable residue field.

Theorem 2.1. Let $(R, \mathfrak{m})$ be a $C M$ local ring with a canonical module. If $R$ has countable CM type, then $R_{\mathfrak{p}}$ has countable CM type for each prime $\mathfrak{p}$ of $R$.

Proof. Let $\mathfrak{p} \in \operatorname{Spec}(R)$ and assume that $\left\{M_{\alpha}\right\}$ is an uncountable family of $R$ modules such that $\left\{\left(M_{\alpha}\right)_{\mathfrak{p}}\right\}$ are nonisomorphic indecomposable MCM $R_{\mathfrak{p}}$ modules. For each $\alpha$ there is a Cohen-Macaulay approximation of $M_{\alpha}$, that is, a short exact sequence

$$
0 \longrightarrow Y_{\alpha} \longrightarrow X_{\alpha} \longrightarrow M_{\alpha} \longrightarrow 0
$$

wherein $X_{\alpha}$ is an MCM $R$-module and $Y_{\alpha}$ has finite injective dimension [3].

Since there are uncountably many modules $X_{\alpha}$, there must be uncountably many $X_{\alpha}$ of some fixed multiplicity. Fixing that multiplicity, and using that there are only countably many isomorphism classes of MCMs, we then find that there are uncountably many short exact sequences

$$
0 \longrightarrow Y_{\beta} \longrightarrow X \longrightarrow M_{\beta} \longrightarrow 0
$$

where $X$ is a fixed MCM $R$-module, $Y_{\beta}$ has finite injective dimension, and the $M_{\beta}$ are among our original list of $M_{\alpha}$.

Since each $\left(M_{\beta}\right)_{\mathfrak{p}}$ is a MCM $R_{\mathfrak{p}}$-module and $\left(Y_{\beta}\right)_{\mathfrak{p}}$ has finite injective dimension over $R_{\mathfrak{p}}, \operatorname{Ext}_{R}^{1}\left(M_{\beta}, Y_{\beta}\right)_{\mathfrak{p}} \cong \operatorname{Ext}_{R}^{1}\left(\left(M_{\beta}\right)_{\mathfrak{p}},\left(Y_{\beta}\right)_{\mathfrak{p}}\right)=0$. This follows from 11, Proposition 4.9]: if $Y$ is a finitely generated module having finite injective dimension, then for all finitely generated $R$-modules $M$,

$$
\operatorname{depth}(M)+\sup \left\{i \mid \operatorname{Ext}_{R}^{i}(M, Y) \neq 0\right\}=\operatorname{depth}(R) .
$$

In particular, each extension $\left(\chi_{\beta}\right)$ splits when localized at $\mathfrak{p}$. This implies that $\left(M_{\beta}\right)_{\mathfrak{p}}$ is a direct summand of $(X)_{\mathfrak{p}}$ for each $\beta$. But over a local ring, a finitely generated $R$-module $Q$ can have at most finitely many nonisomorphic summands 1 Since there are uncountably many $\left(M_{\beta}\right)_{\mathfrak{p}}$ which must be summands of $X_{\mathfrak{p}}$, this contradiction proves the theorem.

\footnotetext{
${ }^{1}$ This can be seen by passing to the completion, where the Krull-Schmidt theorem holds. After completion, any direct summand of $\widehat{Q}$ must be isomorphic to a direct sum of a fixed finite subset of the indecomposable summands of the completion of $Q$. Hence there are only finitely many such isomorphism classes after completion, and since the completions of two modules are isomorphic if and only if the two modules are isomorphic, we are done (see [7. Proposition (2.5.8) Chap. IV, Section 2]).
} 
The results above, together with known examples, suggest a plausible question:

Let $R$ be a complete local Cohen-Macaulay ring of countable CM type, and assume that $R$ has an isolated singularity. Is $R$ then necessarily of finite CM type?

We end the paper with an observation that having countable CM type descends from faithfully flat overrings. The method follows that of [14]. Countable CM type cannot in general ascend to the completion, since countable rings are of countable CM type, but their completions are not of countable CM type if the dimension of the singular locus is at least two. However, when the residue field is uncountable, we do not know if countable CM type ascends to the completion. The proof of the analogous assertion for finite CM type uses that the ring is necessarily Gorenstein on the punctured spectrum, which we do not know is true for countable CM type.

Proposition 2.2. Let $(R, \mathfrak{m})$ be a $C M$ local ring and $(S, \mathfrak{n})$ a faithfully flat $R$ algebra such that the closed fibre $S / \mathfrak{m} S$ is CM. If $S$ has countable CM type, then so does $R$.

Proof. Since the closed fibre is CM, the extension $S \otimes_{R} M$ of any MCM $R$-module is an MCM $S$-module. Let $Z_{1}, Z_{2}, \ldots$ be a complete list of all indecomposable MCM $S$-modules which are direct summands of $S \otimes_{R} M$ for some MCM $R$-module $M$ (ignore any $S$-modules that do not appear in a direct-sum decomposition of some extended module). For each $i=1,2, \ldots$, choose an indecomposable MCM $R$-module $X_{i}$ so that $Z_{i} \oplus W_{i} \cong S \otimes_{R} X_{i}$ for some $S$-module $W_{i}$.

For an indecomposable MCM $R$-module $N$, write $S \otimes_{R} N \cong \bigoplus_{i} Z_{i}^{a_{i}}$, where all but finitely many of the $a_{i}$ are zero. We assume that $a_{i}=0$ for $i>n$ and write the sum as a finite one. Then

$$
\left(S \otimes_{R} N\right) \oplus W_{1}^{a_{1}} \oplus \cdots \oplus W_{n}^{a_{n}} \cong S \otimes_{R}\left(X_{1}^{a_{1}} \oplus \cdots \oplus X_{n}^{a_{n}}\right),
$$

so $S \otimes_{R} N$ is a direct summand of $S \otimes_{R}\left(X_{1}^{b} \oplus \cdots \oplus X_{n}^{b}\right)$, where $b=\max \left\{a_{i}\right\}$. In other words, $S \otimes_{R} N$ is in the "plus category" of $S \otimes_{R}\left(X_{1} \oplus \cdots \oplus X_{n}\right)$ (see [14]). By 14. Lemma 1.2], $N$ is in the plus category of $X_{1} \oplus \cdots \oplus X_{n}$, and by [14. Theorem 1.1], there are only finitely many possible such $N$. Since the set of all finite subsets of $\left\{X_{1}, X_{2}, \ldots\right\}$ is a countable set, this shows that $R$ has only countably many indecomposable MCM modules up to isomorphism.

\section{REFERENCES}

1. M. Auslander, Isolated singularities and the existence of almost split sequences, Proc. ICRA IV, Lecture Notes in Mathematics, vol. 1178, Springer-Verlag, New York-Berlin, 1986, pp. 194241. MR 87j:13029

2. (1986), no. 2, 511-531. MR 87e:16073

3. M. Auslander and R.-O. Buchweitz, The homological theory of maximal Cohen-Macaulay approximations, Mém. Soc. Math. France (N.S.) (1989), no. 38, 5-37, Colloque en l'honneur de Pierre Samuel (Orsay, 1987). MR 91h:13010

4. R.-O. Buchweitz, G.-M. Greuel, and F.-O. Schreyer, Cohen-Macaulay modules on hypersurface singularities II, Invent. Math. 88 (1987), 165-182. MR 88d:14005

5. L. Burch, Codimension and analytic spread, Proc. Camb. Phil. Soc. 72 (1972), 369-373. MR 46:3512

6. G.-M. Greuel and H. Knörrer, Einfache Kurvensingularitäten und torsionfreie Moduln, Math. Ann. 270 (1985), 417-425. MR 86d:14025

7. A. Grothendieck, Éléments de Géometrie Algébrique Chapter IV, 2nd partie, Publ. Math. I.H.E.S. 24 (1965). MR 33:7330 
8. C. Huneke and G. Leuschke, Two theorems about maximal Cohen-Macaulay modules, Math. Ann. 324 (2002), 391-404.

9. H. Knörrer, Cohen-Macaulay modules on hypersurface singularities I, Invent. Math. 88 (1987), 153-164. MR 88d:14004

10. G. Leuschke and R. Wiegand, Ascent of finite Cohen-Macaulay type, J. Algebra 228 (2000), 674-681. MR 2001k:13035

11. P. Roberts, Homological invariants of modules over commutative rings, Seminaire de Mathematiques Superieures 72 (1980), University of Montreal Press. MR 82j:13020

12. F.-O. Schreyer, Finite and countable CM-representation type, Singularities, Representation of Algebras, and Vector Bundles: Proceedings Lambrecht 1985 (G.-M. Greuel and G. Trautmann, eds.), Lecture Notes in Mathematics, vol. 1273, Springer-Verlag, New York-Berlin, 1987, pp. 934. MR 88j:14005

13. R. Y. Sharp and P. Vámos, Baire's category theorem and prime avoidance in complete local rings, Arch. Math. (Basel) 44 (1985), no. 3, 243-248. MR 86h:13004

14. R. Wiegand, Local rings of finite Cohen-Macaulay type, J. Algebra 203 (1998), 158-168. MR 99c:13025

15. Y. Yoshino, Cohen-Macaulay modules over Cohen-Macaulay rings, London Math. Soc. Lect. Notes Ser., vol. 146, Cambridge University Press, 1990. MR 92b:13016

Department of Mathematics, University of Kansas, Lawrence, Kansas 66045

E-mail address: huneke@math.ukans.edu

URL: http://www . math.ukans.edu/ ${ }^{\text {huneke }}$

Department of Mathematics, University of Kansas, Lawrence, Kansas 66045

E-mail address: gleuschke@math.ukans.edu

$U R L:$ http://www. math.ukans.edu/ gleuschke 